\title{
Histoire mondiale du bonheur
}

François Durpaire (dir), Cherche Midi, 2020, 495 p.

\section{Jean-Pierre Véran}

\section{OpenEdition}

\section{Journals}

Édition électronique

URL : https://journals.openedition.org/ries/10010

DOI : 10.4000/ries.10010

ISSN : 2261-4265

Éditeur

France Education international

Édition imprimée

Date de publication : 1 décembre 2020

Pagination : $42-44$

ISBN : 978-2-85420-628-9

ISSN : $1254-4590$

\section{Référence électronique}

Jean-Pierre Véran, «Histoire mondiale du bonheur », Revue internationale d'éducation de Sèvres [En ligne], 85 | décembre 2020, mis en ligne le 01 décembre 2020, consulté le 07 décembre 2022. URL : http:// journals.openedition.org/ries/10010 ; DOI : https://doi.org/10.4000/ries.10010

Ce document a été généré automatiquement le 7 décembre 2022.

Tous droits réservés 


\title{
Histoire mondiale du bonheur
}

François Durpaire (dir), Cherche Midi, 2020, 495 p.

\author{
Jean-Pierre Véran
}

\section{RÉFÉRENCE}

Histoire mondiale du bonheur, François Durpaire (dir), Cherche Midi, 2020, 495 p.

1 Préfacée par Alain Corbin, l'Histoire mondiale du bonheur, parue en février 2020, rassemble les contributions de plus de soixante spécialistes - historiens, philosophes, sociologues, anthropologues, psychologues - réunis pour dresser une fresque historique et planétaire du bonheur, de l'homme de Cro-Magnon à 2100. En cinquante articles regroupés en neuf entrées principales, des bonheurs antiques de Chine, d'Inde ou d'Égypte aux bonheurs sacrés du judaïsme, du christianisme et de l'islam, des bonheurs politiques de la Révolution française et de la révolution chinoise aux bonheurs marchands du rêve américain, des sciences du bonheur anglo-saxonnes aux bonheurs promis par la technologie, ce parcours nous invite à penser une écologie du bonheur, comme le propose François Durpaire en épilogue.

Cette somme ambitieuse permet notamment de poser quelques jalons historiques sur le bonheur en éducation.

Longtemps, on a considéré le bonheur individuel et collectif comme la fin l'éducation, non comme son moyen. Selon Martine Méheut, Aristote accorde, par exemple, un rôle capital à l'éducation pour parvenir au bonheur, l'homme étant son propre artisan, mais il s'agit d'obéir à la raison de l'éducateur et l'éducation reste un dressage. Pour les Athéniens, l'éducation prépare le futur citoyen, par conséquent, apprendre à parler, c'est apprendre à penser, d'où le rôle majeur de la rhétorique dans les études. La découverte du Nouveau Monde conduit à opposer, selon Jean-Paul Duviols, la vie libre et guerrière des indigènes du Brésil à la vie des Européens forgée par une éducation religieuse. Rousseau, d'après Guy Lapostolle, Bruno Jay et Xavier Riondet, associe fortement bonheur et éducation. Pour lui aussi, il s'agit, dans Émile, de faire de l'élève le citoyen non de la société actuelle mais d'une société « dans laquelle l'homme sera à la 
fois libre et heureux ». "Le travail de l'éducateur, nous dit-il, s'il veut rendre le sujet qu'il éduque épanoui et heureux, consiste d'abord, paradoxalement, à le frustrer, ou plus précisément à lui apprendre à accepter la frustration, à faire avec elle sans en pâtir plus qu'il ne faut. »

Si la «recherche du bonheur » constitue le socle de la démocratie américaine selon Marie-Cécile Naves, les révolutionnaires français accordent à l'éducation une place majeure pour réaliser le bonheur public. Au même moment, en Angleterre, William Godwin pense à "créer une génération du bonheur ", comme l'écrit JohnErik Hansson. Godwin propose une pédagogie anti-autoritariste, "anarchisante", visant le bonheur individuel et collectif: " pour que l'espèce [humaine] soit heureuse, il faut non seulement apprendre aux enfants à être heureux individuellement, mais aussi utiles, c'est-à-dire vertueux ». Ainsi, «l'éducation la plus efficace et la plus éthique, écrit Hansson, est celle qui maximise l'autonomie de l'apprenant. " Comme, pour Godwin, "toute éducation est despotisme", il emprunte une voie qui se distingue de celle de Rousseau dans Émile. Il ne s'agit pas de créer des situations artificielles pour faire croire à celui qui apprend qu'il découvre ce qu'on veut lui enseigner, mais au contraire de pratiquer une franchise absolue. Et, pour éviter une emprise trop forte du mentor sur son élève, mieux vaut un enseignement collectif, fondé sur le dialogue, qui ne procède pas par inculcation, mais qui répond à la demande de chaque élève. Laurent Jeannin, Alain Jaillet et Béatrice Mabilon-Bonfils rappellent l'interdiction du travail des enfants à 8 ans et du travail de nuit jusqu'à 13 ans en France en 1841, l'adolescence commençant à devenir une classe d'âge au début de la III ${ }^{e}$ République, avec la scolarité obligatoire jusqu'à 13 ans ; «en cent ans, l'âge moyen de fin d'études est passé de 11,5 à 18,5 ans en France ». La Convention internationale des droits de l'enfant, en 1989, consacre des principes fondamentaux de protection de tous les enfants du monde. Il faut aussi observer comment s'imposent progressivement, au cours $d u \mathrm{XIX}^{\mathrm{e}}$ puis $\mathrm{du} \mathrm{Xx}^{\mathrm{e}}$ siècle, les règles qui vont peu à peu constituer un code de l'hygiène scolaire, en Suisse comme en France, prescrivant des locaux salubres, aérés et bien éclairés (tous les enfants doivent être droitiers avec la lumière naturelle venant de gauche), et un mobilier adapté (tous les enfants ont la même taille, avec les blocs tablebanc).

On s'attarde, avec Fabienne Serina-Karsky, auteure de l'article «Le bonheur à l'école ", sur la révolution de l'éducation nouvelle fondée sur le respect de l'individualité et des rythmes de l'enfant, qui avec Piaget, Wallon, influence l'école dès les années 1920. De Freinet en France à Dewey aux États-Unis, les idées de coopération dans l'apprentissage ou de démocratie de la pédagogie visent à promouvoir le respect du rythme de l'enfant, le droit à l'erreur, l'élaboration de règles en commun, dans un milieu sécurisant. C'est le siècle de l'émancipation de l'enfant dans le respect des autres, via la pratique du selfgovernment. Les écoles nouvelles, d'abord privées, se développent aussi au sein du service public après la Seconde Guerre mondiale, puisant leur inspiration chez Piaget, Vygotski, Dewey, Freud, Montessori, Wallon.

6 L'exemple de l'Équateur, aux $\mathrm{XIX}^{\mathrm{e}}$ et $\mathrm{Xx}^{\mathrm{e}}$ siècles, illustre, selon Emmanuelle Sinardet, la place du bonheur dans l'histoire de l'éducation sud-américaine. Passer d'un État théocratique promu par les conservateurs, qui confient le monopole de l'éducation à l'Église catholique, à sa sécularisation par les libéraux à la fin du XIxe siècle, se traduit, grâce à l'éducation, par la conquête d'une dignité et d'une autonomie bafouées par l'Église, comme par les superstitions. À partir des années 1930, le bonheur n'est plus 
seulement une fin de l'école, mais y entre avec la diffusion des idées de Dewey et Decroly, qui promeuvent un élève acteur de ses apprentissages. À la fin du $\mathrm{xx}^{\mathrm{e}}$ siècle, les mouvements amérindiens revendiquent des droits politiques, économiques et culturels, battant en brèche l'uniformisation par la langue et la culture dominante hispanocoloniale. L'éducation a été l'instrument de l'homogénéisation culturelle sur le modèle colonial, mais le bonheur désormais change de sens : il s'agit de vivre en harmonie avec son environnement et non plus de le dominer...

7 De ce rapide survol, on pourra retenir le fait que progressivement, l'éducation n'est plus seulement la condition du bonheur futur, mais que le bonheur de celles et ceux qu'on éduque est la clé de leur réussite dans les apprentissages. On retiendra aussi combien des peuples longtemps méprisés sur les continents colonisés ont une conception du bonheur beaucoup plus adaptée aux enjeux du xxI siècle, parce que l'importance de la relation aux autres, de l'harmonie avec la nature ou de la culture du rêve sont autant de clés pour faire face aux défis actuels. Après avoir prétendu les mettre à notre école de la performance, il est urgent de les écouter et de s'inspirer de leur conception $\mathrm{du}$ bonheur et de l'apprentissage. On s'attardera, grâce à El Hadj Hamidou Kassé, sur l'importance de la relation aux autres et du partage chez les Peuls et, grâce à Fabrice Delsahut, sur la sagesse de la gaieté des Indiens d'Amérique du Nord, ou encore sur le dépassement de l'horizon, constitutif de l'imaginaire du bonheur des Touaregs, selon Hélène Claudot-Hawad.

8 Ceux qui s'intéressent à l'éducation seront sensibles à l'apport, souligné par François Durpaire dans son Épilogue, de chacun des continents à la définition du bonheur: l'Afrique et le "partage», l'Amérique et l'«harmonie», l'Asie et la «sagesse », l'Europe et le "plaisir ", l'Océanie et le «rêve ». Ils retiendront aussi la nécessité d'une « réorganisation des enseignements autour des valeurs humaines et des compétences de vie». Sans doute la crise sanitaire mondiale de 2020, qui a fermé les écoles à deux milliards d'élèves, peut-elle accréditer l'urgence de cette transformation.

\section{AUTEUR}

\section{JEAN-PIERRE VÉRAN}

Jean-Pierre Véran, inspecteur d'académie $(\mathrm{H})$, membre du comité de rédaction de la Revue internationale d'éducation de Sèvres, expert auprès de France Éducation international en coopération éducative et membre professionnel du laboratoire Bonheurs de CY Cergy Paris Université. Auteur de plusieurs ouvrages, il tient également un blog consacré à l'éducation sur Mediapart : http://blogs.mediapart.fr/blog/jean-pierre-veran/ Courriel : jeanpierreveran2[at]gmail.com 